\title{
Ptychography with a Virtually Enlarged Illumination.
}

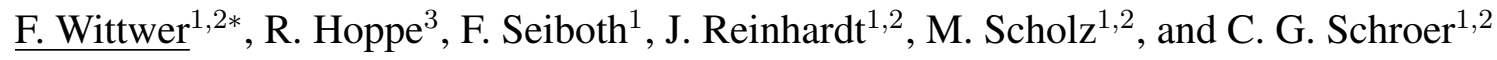 \\ ${ }^{1}$ Deutsches Elektronen-Synchrotron, Hamburg, Germany \\ ${ }^{2}$ University of Hamburg, Department Physik, Hamburg, Germany \\ ${ }^{3}$ German Aerospace Center, Dresden, Germany \\ ${ }^{*}$ Corresponding author: felix.wittwer@ desy.de
}

Ptychography has become a key tool for high-resolution X-ray microscopy. We present here an extension to it that allows to work with insufficiently sampled diffraction patterns and reconstructs previously unseen parts of the sample. In ptychographic imaging the sample is scanned through a coherent beam recording the intensity distribution of the transmitted scattered wavefield at each scanpoint. Based on the ensemble of diffraction patterns and redundant information in adjacent diffraction patterns, ptychography retrieves the complex-valued transmission function of the sample. At the same time, also the illuminating probe is reconstructed in amplitude and phase. Due to this, the ptychography reconstructions are independent of any aberrations from the optics and the spatial resolution is not limited by the numerical aperture of the optics. Today, resolutions in the hard X-ray regime of $30 \mathrm{~nm}$ down to $10 \mathrm{~nm}$ are routinely achieved. One of the most popular ptychographic algorithms, ePIE [1], now has many extensions to deal with different experimental conditions, for example for thick, non-planar objects a multislice variant was proposed [2]. One extension concerns the influence of the detector on the measured diffraction patterns. A detector pixel integrates the number of photons over its area. A speckle can only be properly sampled, if it extends over multiple pixels. When the speckles are of the same size or smaller than a pixel, the sampling theorem is not fulfilled resulting in loss of information that leads to artefacts in the reconstruction. The up-sampling ptychographic iterative engine (sPIE), however, is able to reconstruct sample and probe even when the speckle are smaller than one pixel [3]. Due to the reciprocal relationship between distances in the sample plane and the detector plane and accordingly between the size of the probe and the resulting speckles, small speckles correspond to a large probe and vice versa. We demonstrate that sPIE can be used for aberrated focusing optics, which produce a larger-than-designed probe, and that this larger probe allows for the reconstruction of hitherto unseen parts of the sample outside of the original field of view.

The experiment was carried out at the Nanoprobe endstation of beamline P06 at PETRA III, DESY, Hamburg. Two crossed refractive lamellar lenses (RLL) [4] were used to focus the $15.25 \mathrm{keV}$ X-ray beam to a spot size of $150 \mathrm{~nm} \times 200 \mathrm{~nm}$. To characterize the lenses with ptychography, a Siemens star test pattern from NTT-AT was scanned across the focused beam. The scan covered an area of $2.5 \mu \mathrm{m} \times 2.5 \mu \mathrm{m}$ in $38 \times 38=1444$ equidistant positions with a step size of $67 \mathrm{~nm}$. A LAMBDA photon detector (XSpectrum $\mathrm{GmbH}$ ) was placed $2.16 \mathrm{~m}$ behind the sample to capture the diffraction patterns. The patterns were cropped to $256 \times 256$ pixels. With a detector pixel size of $55 \mu \mathrm{m}$, this results in a real-space pixel resolution of $12.5 \mathrm{~nm}$ in the ptychographic reconstruction. The size of the computational window of the reconstructed probe follows directly by multiplying the resolution with the number of pixels, giving $3.2 \mu \mathrm{m}$. Aberrations in the RLLs caused the focused beam to have strong tails. These tails increased the focus size to more than $4 \mu \mathrm{m}$ diameter. Since the tails from the aberrations extended beyond the computational window of the probe, the diffraction patterns consequently could not be fully resolved by the LAMBDA detector.

Applying the standard ePIE algorithm on such data sets typically results in images as shown in Fig. 1a) 

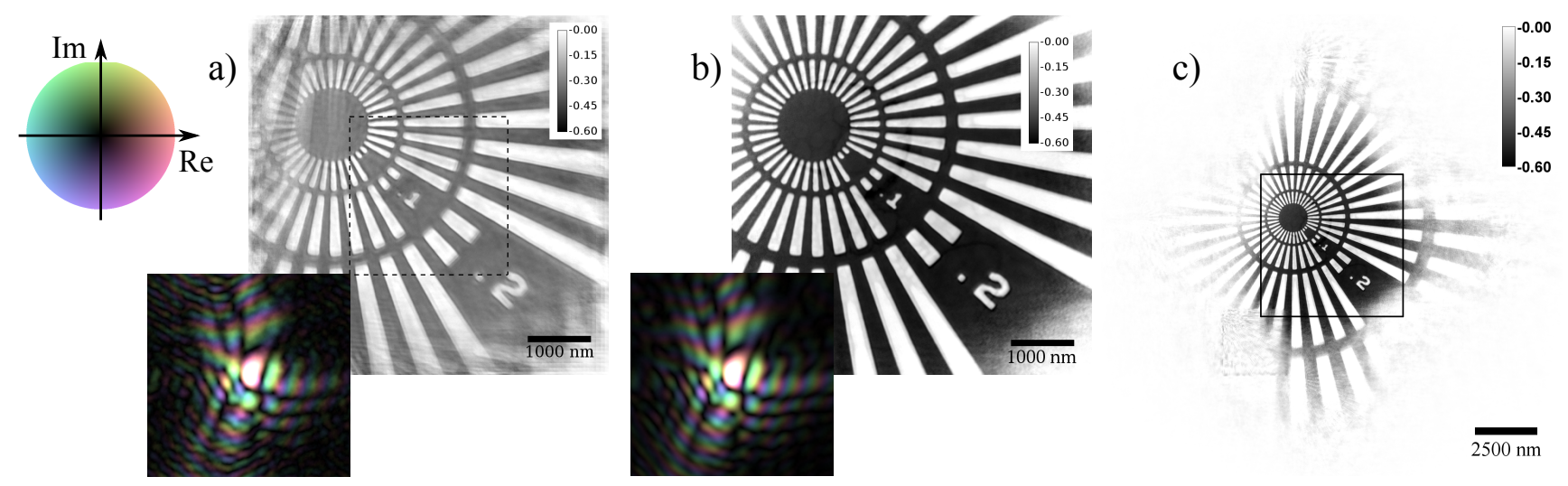

Figure. 1. a) Reconstructed object phase shift and complex probe using ePIE; the scanned area is indicated by the dashed square and the probe contrast enhanced by a factor 3. b) Central part of the sPIE-reconstructed object phase shift and the central part of the probe, with the same field of view and scaling as a). c) Entire reconstructed object phase shift using sPIE, the square marks the field of view in a) and b).

revealing pronounced reconstruction artifacts. The dashed square marks the $2.5 \mu \mathrm{m} \times 2.5 \mu \mathrm{m}$ scanned area. The object reconstruction stretches on all four sides up to the edge, which is a clear sign that the illumination is too large. The obvious correctives, using a detector with smaller pixels or increasing the distance between the sample and the detector are often not feasible due to practical limitations and the given experimental conditions. Instead, we used sPIE to use a virtual detector with smaller pixels.

We divided each real pixel into $5 \times 5$ virtual subpixels, thus increasing the maximum probe size five times to $16 \mu \mathrm{m}$. Fig. 1 compares the reconstruction results from ePIE and sPIE. Fig. 1b) shows the same area of the object and illumination as a), but reconstructed with sPIE. The illumination in a) wraps around vertically and interferes with itself, leading to horizontal stripes. This is no longer the case for the virtually enlarged illumination which is smoother. The numerical artifacts in the object shown in Fig. 1a) are considerably reduced and the contrast has strongly improved. The reconstruction still extends to the edge, but with the increase of the illumination size, the size of the reconstructed object naturally also grows, here from $5.7 \mu \mathrm{m}$ to $18.5 \mu \mathrm{m}$. Thus, b) shows only the central part and c) provides the full reconstruction. Strikingly, the object reconstruction now extends beyond this border and previously concealed parts of the sample become visible. The Siemens star contains rings that mark certain structure sizes, $0.1 \mu \mathrm{m}, 0.2 \mu \mathrm{m}$, etc. The smallest, marking $0.1 \mu \mathrm{m}$, is fully visible in all reconstructions. The next bigger one, for $0.2 \mu \mathrm{m}$, is only partially visible in the ePIE reconstruction, but is completely reconstructed using sPIE. Additionally, the even larger $0.5 \mu \mathrm{m}$ ring is reconstructed in segments.

In conclusion, we have shown that virtual subpixels are valuable to reconstruct unknown aberrated optics with enlarged probe size. At the same time, the extension of the field of view of the reconstructed object removes artefacts and accelerates the scanning of large areas with high resolution.

References:

[1] AM Maiden and JM Rodenburg, Ultramicroscopy 109 (2009) 1256-1262.

[2] AM Maiden, MJ Humphry and JM Rodenburg, JOSA A 29 (2012) 1606-1614.

[3] DJ Batey, et al., Phys. Rev. A 89 (2014) 043812.

[4] F Seiboth, et al., Appl. Phys. Lett. 105 (2014) 131110. 